\title{
Grammatical Gender in the Production of Single Words: Some Evidence from Greek
}

\author{
Evangelia Plemmenou, Ellen G. Bard, and Holly P. Branigan \\ University of Edinburgh, Edinburgh, Scotland, United Kingdom
}

Published online November 20, 2001

\begin{abstract}
The present study investigated the effect of prior grammatical gender information provided by the production of a bare noun on the production of a syntactically unrelated, gender-inflected color adjective. The target language was Greek. A lexical priming task involving picture naming was employed. Participants saw a series of pictures, some in color and some in blackand-white; they had to name a black-and-white picture with the single noun (prime) and a color picture with the appropriately inflected color adjective (target). Prior gender information was shown to affect subsequent production of gender-marked words. The effect was restricted to nouns of one gender class (masculine) only. The implications of these results for the representation and processing of gender in production are discussed. ๑ 2001 Elsevier Science (USA)
\end{abstract}

Grammatical gender is a syntactic property of words; its representation, retrieval, and use have been examined in a number of recent studies (e.g., see Friederici \& Jacobsen, 1999, for a review of grammatical gender in word recognition; Schriefers \& Jescheniak, 1999, for a review of grammatical gender in word production) with respect to the functional architecture and the retrieval mechanisms of the mental lexicon (e.g., Caramazza, 1997; Levelt, Roelofs, \& Meyer, 1999). Some of the critical questions addressed have been, first, the nature of the lemma-to-gender connection, i.e., whether it is unidirectional or bidirectional; second, the linguistic contexts in which gender information is assumed to be selected, i.e., in the presence or not of an agreement target in the noun's local syntactic environment; and, third, the effect (if any) on gender processing of overt phonological markers. Thus far, variable results have been obtained as a result of task and/or language manipulations.

The present study employed a production task to investigate gender priming, i.e., whether preactivation of a word's gender can facilitate production of that word. Related to this, two further questions were addressed: First, whether production of a single noun (with inherent gender) involves retrieval of its gender feature in a way that is reflected in subsequent production of gender-marked words; and, second, whether gender priming can be interpreted as being intralexical, i.e., as affecting the processing characteristics of words, of the same or of different category in the mental lexicon, merely as a function of a shared property (gender) and independent of higher level syntactic structure. Thus far, effects of gender information on word production have been investigated primarily in naming experiments that use extensions of the picture-word interference paradigm or that manipulate the gender (in-)congruency of prior phase/sentence structure information relative to an upcoming word (i.e.,

Address correspondence and reprint requests to Evangelia Plemmenou, Sokion 16, Nea Elevetia 162 31, Athens, Greece. 
where there is a structural relation between prime and target). Furthermore, in most of the languages examined, gender does not have an overt realisation on the noun (e.g., Dutch) or it is phonologically, but not morphologically marked (e.g., Italian, Spanish, and Catalan). Recent findings have pointed to the importance of accounting for the different mapping relations between gender and gender-marked lexical elements, between as well as within languages, as a critical determinant of the effects obtained.

Before turning to the theoretical framework, we provide a brief overview of the Greek gender system. Greek has three genders: masculine, feminine, and neuter. Nouns inflect for gender, which constitutes one of the basic categories underlying the morphology of the Greek nominal system. Nouns are assigned to gender classes largely on the basis of morphological rules. Reference to more than one word forms is made. Thus, for example, nouns ending in the nominative singular -as and in the accusative singular - a are masculine, whereas nouns ending in the nominative singular -as and in the accusative singular -as are neuter. Gender in Greek is marked fusionally with case (structurally conditioned) and with number (largely conceptually conditioned). Gender-by-case-by-number interactions result in variations in the mappings between gender and its inflectional manifestation. Gender agreement is marked on a wide range of elements, e.g., definite and indefinite articles, demonstrative, interrogative, relative pronouns, adjectives, and past participles. Stems in Greek are bound; they only project to the word level if an inflectional suffix is attached to them. Therefore, grammatical gender is a prominent feature of the language as it surfaces morphologically every time a single word is produced.

In line with one of the most prominent models of language production (Roelofs, 1992; Levelt et al., 1999), we assume that the lexical knowledge is organized in three distinct strata: conceptual, syntactic, and morphophonological. Words at the syntactic stratum are represented by lemma nodes. Each lemma node connects to nodes representing the word's syntactic properties. The types of information specified at this level include major category information (e.g., noun and adjective), featural information (e.g., gender, case, number for nouns, number, person, tense, and tense for verbs), and combinatorial information. All nouns and agreement targets, e.g., determiners and adjectives, of the same grammatical gender are connected to one shared gender node. Nodes at each layer are assumed to be accessed serially so that processing at the lemma level strictly precedes processing at the morphophonological level. When a lemma node is activated, the nodes linked to it are also assumed to be activated.

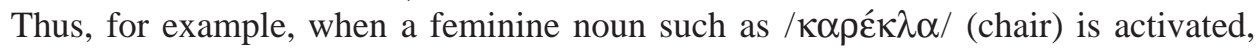
the corresponding gender node alone with, e.g., the syntactic category, number, and case nodes are also activated. Importantly, a node can be activated without being selected.

Under Levelt et al.'s account, lemma node retrieval is always implicated in the production of words, independent of the syntactic context in which they occur (but see Caramazza, 1997, for an alternative account). However, the selection of the syntactic nodes associated with a given lemma is dependent on the syntactic context: in the production of a single noun, its gender feature is merely activated. It will be

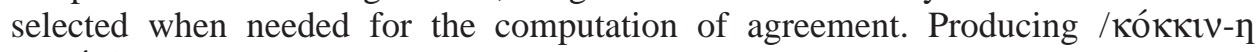
$\kappa \alpha \rho \varepsilon ́ \kappa \lambda-\alpha /$ (red chair) does not demand selection of the node for feminine gender, since in this context, the adjective has to agree with the noun it modifies. Gender priming should only be obtained in producing gender-marked utterances.

In a language like Greek, even single-noun utterances are gender marked. As noted above, not only do words bear inflectional markers of syntactic attributes (with grammatical gender being one of them), but also they do so invariably, since bare stems cannot qualify as words in the language. Hence, it can be argued that in Greek, single- 
noun production involves selection both of the noun's lemma node and of its associated syntactic specifications. Each of these nodes will subsequently retain some residual activation. If there is a single gender node shared by all words of that gender, subsequent production of single words sharing the same gender should be facilitated, giving rise to priming effects.

Of course, production of a phrase also requires selection of gender information so that word categories other than nouns, such as determiners or adjectives, will bear the appropriate gender markers. A critical distinction between nouns and the latter two categories with respect to their lexical reprsentation is that for nouns, gender is an inherent property: A given noun is masculine, feminine, or neuter irrespective of its syntactic environment. For determiners and adjectives gender is a noninherent property and conditioned structurally. Levelt et al.'s model assumes that words with inherent and noninherent gender alike are linked to a common gender node. It therefore predicts that both the production of single nouns and of determiners or adjectives should involve the same gender node. Thus, when a masculine noun is produced, the retrieval of masculine gender leaves residual activation of the gender node so that reselection of the same gender node in the production of, e.g., a masculine adjective will be facilitated.

We tested these predictions using a lexical priming task in which participants pro-

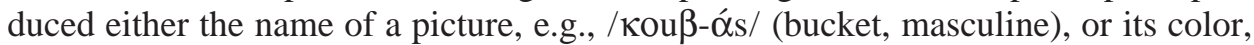
e.g., / $\pi \rho \alpha \dot{\sigma} \sigma \mathrm{v}-\mathrm{os} /$ (green, masculine). The gender of the noun prime was either the same as or different from the gender of the adjective target. If a common gender node subserves the production of both nouns and adjectives, and if this node is accessed during the production of single nouns, then we expect that production of a target adjective will be faster following a same gender noun prime than following a different gender noun prime.

\section{METHOD}

\section{Procedure and Design}

Participants were tested individually on a Macintosh computer using the PsyScope experimental program. In the preview block, all pictures (prime, target, and filler) were presented in black-and-white for $2 \mathrm{~s}$, after which the name appeared underneath the picture. At this stage, no overt response was required. In the main experiment, participants were instructed to produce either a gender-inflected color adjective

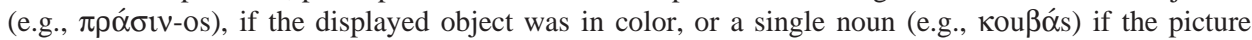
was black-and-white; they were asked to respond both quickly and accurately and to try to use the names they had seen during the preview. Following a short practice session of 15 trials, the experimental trials began. Each trial was structured as follows: a visual warning signal appeared for $600 \mathrm{~ms}$ followed by a pause of $200 \mathrm{~ms}$. Then the picture was displayed, which remained on the screen until participants responded, for a maximum of $2000 \mathrm{~ms}$. The screen was cleared for $1600 \mathrm{~ms}$ before the next trial was initiated.

\section{Participants}

Participants were 18 native speakers of Greek enrolled at the University of Edinburgh.

\section{Materials}

Target stimuli consisted of 40 pictures of simple objects, 20 of which depicted masculine nouns. The other 20 were feminine nouns. Since frequency statistics for words in Greek were not publicly available at the time the experiment was designed, age-of-acquisition (AoA) ratings for these words were used instead. Of the masculine nouns, 3 were low acquired (LA) and the remaining 17 were medium acquired (MA), while of the feminine nouns, 7 were LA and 13 MA. An additional set of 40 pictures of masculine and feminine nouns were selected to serve as primes in the trials immediately preceding target presenta- 
tion. Prime items also belonged to the low and medium AoA ranges. Sixteen masculine and 14 feminine names were MA, while the remaining 4 and 6 respectively were LA. Prime and target names were controlled for word length. A further set of 40 neuter items were used as fillers in the trials intervening between two consecutive prime-target pairs. Half of the neuter nouns were MA and half were LA. A further 15 pictures from the three gender classes were selected to serve in the practice trials. Every target picture appeared once in each priming condition; each participant saw each picture in one condition only and saw an equal number of pictures in each condition. Primes and targets were semantically and phonologically unrelated. Order of presentation was randomized. Each session contained 120 naming trials. Stimuli were presented in a single block. Pictures were presented in black-and-white or in one of four colors (red, green, yellow, and light blue). Target stimuli were always in color and therefore required gender-inflected adjective responses. Primes, on the other hand, were always black-and-white pictures, thus eliciting single-noun responses. The fillers were presented half in black-and-white and half in color. For the priming condition targets were immediately preceded by primes of the same gender, while for the unprimed condition, targets were immediately preceded by primes of a different gender. The experiment lasted approximately $20 \mathrm{~min}$.

\section{RESULTS}

Two-way analyses of variance (ANOVAs) were carried out on target onset times and error data with prime type (same-gender or different-gender from target; within participants, within items) and target gender (masculine or feminine; within participants, between items) as the two variables. Two items, one masculine and one feminine, were excluded from the analysis due to high error rate. Target responses were also removed prior to the analyses if erroneous in one of the following ways: the (colored) picture was named with a noun instead of an adjective (2.5\%); the adjective was incorrectly inflected $(5.5 \%)$; a wrong color adjective $(.3 \%)$ or wrong prime was produced $(.5 \%)$; or the response began with a hesitation, was interrupted, or was initiated too late, i.e., after $2000 \mathrm{~ms}$ from picture onset (4.2\%). Thirteen percent of the data was treated in this way. Furthermore, response times that deviated by more than 2.5 SD from the mean of the subject or the item were replaced by the subject or the item mean, $\pm 2.5 S D$, respectively (0.016). Table 1 presents mean RTs in milliseconds for the target stimuli, number of correct responses, and error percentages as a function of prime and target gender.

Analysis of the target responses revealed a significant main effect of target gender by participants, $F 1(1,17)=9.810, p<.05$. It also revealed a significant prime gender by target gender interaction, with both participants and items, in line with the gender priming hypothesis, which had predicted faster gender marked target responses such as / KóкKıv-os/ (red, masculine) after a same-gender prime response

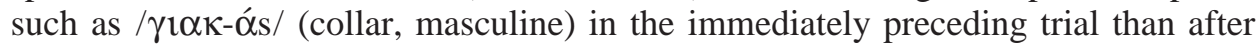
a different-gender response such as / $\tau$ oúp $\tau-\alpha /$ (cake, feminine), $F 1(1,17)=7.227$, $p<.05, F 2(1,36)=4.109, p .05$. The observed discrepancy in the effect of priming ( $75 \mathrm{~ms}$ for masculine words but only $12 \mathrm{~ms}$ for feminine words) was confirmed by planned comparisons between primed and unprimed responses of masculine $(t 1=$

\section{TABLE 1}

Mean Target Utterance Onset Latencies (in Milliseconds), Number of Valid Responses and Error Percentages as a Function of Prime and Target Gender

\begin{tabular}{lcccr}
\hline & $\mathrm{mm}$ & $\mathrm{mf}$ & $\mathrm{fm}$ & $\mathrm{ff}$ \\
\hline Means & 1188 & 1164 & 1263 & 1152 \\
Correct responses & 146 & 150 & 145 & 154 \\
Error \% & 14.7 & 12.3 & 15.2 & 10 \\
\hline
\end{tabular}


$-3.678, p<.05 ; t 2=-2.362, p<.05)$ and feminine nouns $(t 1=.296, p>.05$; $t 2=.258, p>.05)$. Analysis of feminine nouns yielded no evidence of facilitation over either participants or items. The absence of any significant correlation between AoA scores and response latency-primed RTs $(r=.232, p>.05)$ or unprimed RTs $(r=.093, p>.05)$ excluded the possibility that the different pattern of results for the two noun classes could be attributed to differences in the somewhat different distribution of masculine and feminine items in the medium and low AoA ranges. Masculine adjectives, primed and unprimed, induced longer RTs than feminine adjectives. However, no significant correlation was obtained between response latencies and priming effect (unprimed-primed): $r=.225, p>.05$. ANOVAs performed on the error data revealed no significant main effect of interaction with either participants or items $(F \mathrm{~s}<1)$.

\section{DISCUSSION}

The present study aimed at investigating whether prior grammatical gender information provided by the production of a single noun could affect the production of a gender-inflected adjective. Three related issues addressed were, first, whether grammatical gender is accessed even when such information is not necessary; second, whether gender priming can be obtained when prime and target are syntactically unrelated and belong to different word categories (nouns and adjectives); and, third, whether gender priming can be interpreted as occurring within the lexicon, temporarily modifying the processing characteristics of words, independent of higher level syntactic relations.

The absence of a gender priming effect for feminine nouns, compared with the reliable effects obtained for masculine nouns, does not provide clear evidence as to the role of grammatical gender in the context discussed. The results from the masculine items alone supported the gender priming hypothesis, which predicted faster

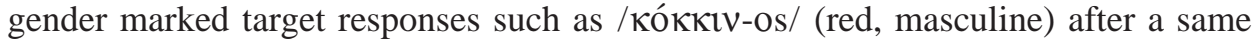
gender prime response such as / $\gamma 1 \alpha \kappa$-ós/ (collar, masculine) in the immediately preceding trial than after a different gender response.

Although the production of a single noun is assumed not to require gender information, this does not necessarily imply that speakers can avoid automatic access to the noun's gender. The spreading activation architecture of the Levelt et al. model of the mental lexicon assumes gender access as a mere side effect of lemma node selection. The presence of gender priming as a result of single (masculine) noun production is in line with this assumption. As noted above, however, nouns in Greek are gender marked. Given the seriality assumption of the Levelt et al. model, processing at the lemma level is completed before processing at the morphophonological level is initiated. Therefore, the presence or not of morphophonological gender markers and the properties of the latter, e.g., distribution, frequency, transparency or opacity, and (multi-)functionality, should not affect the way gender is processed at an abstract lexical-syntactic level of representation. However, it should be noted that studies thus far (particularly from picture-word interference tasks) have not established the precise locus of gender competition (or facilitation), i.e., at syntactically or phonologically specified levels of representation. Furthermore, if an alternative to the seriality processing assumption is adopted, of the type suggested by cascaded or interactive processing models of word production, different predictions are derived. It is therefore an empirical issue whether the morphological realization of gender has an effect on the way gender, as an abstract lexical-syntactic property, is processed and whether gender retrieval is invariably implicated in the production of single nouns irrespective of whether they are gender-marked. 
In the present task, primes and targets belonged to different word categories and were not embedded in phrasal or sentential contexts. The effect obtained for masculine nouns is compatible with the idea of gender priming as an intralexical effect, i.e., an effect occuring within the lexicon, independent of higher level syntactic relations between prime and target. It appears that gender priming can be obtained for syntactically unrelated primes and targets, while still maintaining the assumption of a unidirectional lemma-to-gender node. Particularly, gender priming is thought of as the result of facilitated reaccess to a gender node rather than the result of unconstrained spreading of activation to all gender matching elements in the lexicon. If word production involves the selection of a gender node, it will be speeded up as a function of residual gender node activation due to earlier selection.

The observed discrepancy in the pattern of results for masculine and feminine nouns does not allow for a unified treatment of the role of gender in the present context, while asking for further exploration of the effects. The distribution of masculine and feminine nouns in the AoA ranges could not account for this difference. In fact, there was no significant correlation at all between AoA scores and response latencies. This is somehow surprising given that AoA scores are held to be better predictors of response latencies in picture naming than frequency measures. The two issues seem to be at hand here: first, whether the locus of the AoA effect is at the level of lemmas or at the level of lexemes (frequency effects have been located at the latter level) and, second, whether the production of adjectives (an instance of anaphoric lexical access) involves access to both the noun's lemma and lexeme specifications. Two further questions which warrant exploration in future research refer to the properties of morphological markers (e.g., frequency and distribution) and to the role of inflectional class membership as part of the syntactic specification of nouns.

\section{REFERENCES}

Caramazza, A. (1997). How many levels of processing are there in lexical access? Cognitive Neuropsychology, 14(1), 177-208.

Friederici, A., \& Jacobsen, T. (1999). Processing grammatical gender during language comprehension. Journal of Psycholinguistic Research, 28(5), 467-484.

Levelt, W. J. M., Roelofs, A., \& Meyer, A. (1999). A theory of lexical access in speech production. Behavioural and Brain Sciences, 22, 1-75.

Roelofs, A. (1992). A spreading-activation theory of lemma retrieval in speaking. Cognition, 42, 107142 .

Schriefers, H., \& Jescheniak, J. (1999). Representation and processing of grammatical gender in language production: A review. Journal of Psycholinguistic Research, 28(6), 575-600. 